\title{
Evaluación comparativa del nivel de Desarrollo Orientado al Transporte (DOT) en torno a nodos de transporte de grandes ciudades: métodos complementarios de ayuda a la decisión
}

\author{
Francisco-Sergio Campos-Sánchez. Universidad de Granada, Granada, España. \\ Francisco-Javier Abarca-Álvarez. Universidad de Granada, Granada, España. \\ Gloria Serra-Coch. Architecture, Energy and Environment, Barcelona, España. \\ Charlotte Chastel. Ingeniería Urbana, París, Francia.
}

RESUMEN | El Desarrollo Orientado al Transporte (DOT) es un enfoque de la planificación que promueve el desarrollo sostenible de un área urbana. Evaluar el nivel DOT resulta interesante para determinar la idoneidad de los desarrollos urbanos al respecto, así como su potencial de mejora. El objeto del trabajo consiste en evaluar el nivel DOT de importantes áreas urbanas mediante dos métodos diferentes: índice global y análisis multivariable mediante redes neuronales artificiales. Los métodos se contrastan entre sí, clarificando sus límites y grado de complementariedad. Adicionalmente, los resultados se verifican mediante el análisis espacial sig de un caso concreto. Según resultados: la evaluación mediante índice es limitada; el análisis multivariable determina perfiles de áreas semejantes, independientemente de sus índices globales; el análisis espacial pormenoriza los valores medios obtenidos. Estos métodos aportan conocimiento en sus respectivos campos. Si se usan complementariamente entre sí, mejoran su efectividad como herramientas de análisis útiles en planificación.

PALABRAS CLAVE | indicadores ambientales, morfología urbana, planificación urbana. ABSTRACT | Transit Oriented Development (TOD) is a planning approach that promotes sustainable development of an urban area. Assessing the levels of TOD may allow determining the appropriateness of urban development in this regard, as well as its potential for improvement. The purpose of this work is to assess the levels of TOD using two different methods: Global Index and Multivariate Analysis based on Artificial Neural Networks. The methods contrast each other, clarifying both their limits and complementarity degree. Additionally, the results are verified through GIS spatial analysis applied on a case study. According to the results, the Global Index Assessment is limited; the multivariable analysis shows profiles of similar urban areas, regardless of their global indexes; the spatial analysis details the average values obtained. These methods provide knowledge in their respective fields. If used in a complementary way, they improve their effectiveness as useful analysis tool for urban planning.

KEYWORDS | environmental indicators, urban morphology, urban planning.

Recibido el 26 de septiembre de 2017, aprobado el 20 de marzo de 2018

E-mails: F. S. Campos-Sánchez: scampos@ugr.es | F. J. Abarca-Álvarez: fcoabarca@ugr.es | G. Serra-Coch: gloriaserracoch@gmail.com; C. Chastel: charlotte.chastel@eivp-paris.fr 


\section{Introducción}

El Desarrollo Orientado al Transporte (TOD, por sus siglas en inglés) es un conocido enfoque de la planificación que promueve el desarrollo urbano sostenible mediante la integración del transporte público, la densidad, la compacidad, la intermodalidad y los usos mixtos en un área cercana fácilmente caminable y ciclable (Calthorpe, 1993; Cervero, 1998; Cervero, Ferrell \& Murphy, 2002; Center for Transit-Oriented Development [стор], 2009; Dittmar \& Ohland, 2004; Newman \& Kenworthy, 1999), lo que es reconocido como deseable. Este tipo de planificación no debe estar solo orientado a la creación de nuevos desarrollos. También debe pretender la evaluación de entornos urbanos ya construidos que originariamente no fueron pensados desde un enfoque TOD (Singh, Fard, Zuidgeest, Brussel \& van Maarseveen, 2014). Esto es importante para revisar el modo en que se ha resuelto la ciudad en torno a sus principales nodos de transporte, lo que cobra especial relevancia cuando se trata de grandes ciudades en las que decisiones de este tipo repercuten sobre una gran población.

En el mundo académico, la mayor parte de la literatura existente sobre TOD se ocupa solo de la evaluación de algunos de sus aspectos, ya sea teóricamente (Bishop, 2015; Baldwin \& Lombardi, 2004; Renne \& Wells, 2002) o desde un enfoque más práctico, basado en la experiencia acumulada (Instituto de Políticas para el Transporte y el Desarrollo [The Institute for Transportation and Development Policy, ITDP], 2013; Jacobson \& Forsyth, 2008; Suzuki, Cervero \& Iuchi, 2013), pero escasamente lo hace del conjunto de factores que intervienen a la vez. En esta última línea existen trabajos que señalan la necesidad de una evaluación integral del nivel TOD de un entorno construido, mediante una valoración global (Evans, Pratt, Stryker \& Kuzmyak, 2007; Schlossberg \& Brown, 2004; Singh et al., 2014). Esta valoración o Índice TOD, además de cuantificar los criterios manejados de forma espacialmente explícita e integrada, permite comparar áreas diferentes entre sí, lo que ayuda a la toma de decisiones en la planificación.

Es conocido que las inversiones públicas en infraestructura se realizan a menudo sin comprender bien la situación preexistente y sin prever adecuadamente las consecuencias posteriores. En esto no es una excepción la planificación TOD, dada la falta de valoraciones conjuntas al respecto (Renne \& Wells, 2005). Por lo tanto, medir los niveles TOD de un área urbana de forma conjunta ayuda a su puesta a punto. Sin embargo, usar únicamente el Índice Tod para ello no es la panacea. Se trata de un proceso de evaluación complejo en el que intervienen múltiples variables con valoraciones independientes. Aunque varias de estas áreas presenten un Índice TOD idéntico, la semejanza entre ellas a varios niveles es, cuando menos, cuestionable. En la práctica, este tipo de evaluación es orientativo y resulta necesaria su verificación mediante otras técnicas complementarias para validarlo como enfoque útil en la planificación. Una de estas técnicas es el análisis multivariable mediante red neuronal artificial basada en mapas autoorganizados (Self-Organizing Maps o sом), que permite analizar y visualizar conjuntos de indicadores estadísticos para diversas aplicaciones (Kaski \& Kohonen, 1996). Permite, además, clasificar sin asignar a priori una etiqueta con atributos, reduciendo la complejidad de los datos (Spielman 
\& Thill, 2008). La interacción entre las variables se traduce finalmente en agrupaciones, o perfiles de ellas, con aporte de conocimiento asociativo.

Otra de las técnicas complementarias utilizable se basa en el análisis espacial mediante Sistemas de Información Geográfica (SIG). En el ámbito de la movilidad en general y de los desarrollos TOD en particular, se ha afirmado que estas tecnologías significan la apertura a nuevas posibilidades en el análisis de los desarrollos actuales y futuros para afrontarlos con objetivos TOD (Ackerson, 2005; Leslie et al., 2007). La representación espacial de los valores mediante sIG puede completar la representatividad limitada de los resultados numéricos y estadísticos obtenidos (Abarca-Álvarez, Campos-Sánchez \& Reinoso, 2017; Skupin \& Agarwal, 2008; Yeh, 2005), facilitando así su comprensión.

El objeto de este trabajo consiste en evaluar el nivel TOD de entornos construidos (áreas TOD) alrededor de nodos de transporte de grandes ciudades mediante diferentes métodos de evaluación, así como verificarlos y contrastarlos entre sí para determinar su grado de complementariedad o superposición. Esta información puede ser útil a la toma de decisiones dentro de la planificación para elevar los niveles TOD alrededor de estos nodos y unificar los criterios de actuación necesarios para ello. Los métodos de evaluación empleados son: i) Índice de valoración global TOD; y ii) Análisis multivariable del tipo som. Un tercer análisis de tipo espacial mediante SIG va a servir para validar y pormenorizar los resultados derivados de los métodos anteriores. Esto va a permitir verificar la validez del sistema de evaluación basado en índices, así como distinguir en qué aspectos ha de ser matizado y completado.

La metodología empleada consta de las siguientes fases: 1) Recopilación de datos y aplicación de batería de indicadores mediante formulario; 2) Evaluación del nivel TOD mediante Índice TOD; 3) Evaluación del nivel TOD mediante análisis som; y 4) Representación espacial mediante análisis sIg. Finalmente, en el apartado discusivo se interpretan los resultados y se contrastan entre sí, alcanzándose conclusiones relevantes al respecto. Nótese que, basándose en el formulario y el tipo de procesamiento de datos empleados, esta metodología podría ser aplicable a áreas TOD de otros nodos y ciudades similares a las estudiadas.

El caso de estudio se centra en los entornos construidos alrededor de 31 nodos de transporte de carácter central pertenecientes a cinco grandes ciudades: Londres, París, Los Ángeles (LA), Santiago de Chile (Santiago) y Estambul. Todas ellas presentan una gran población y una red de transporte diversa y "madura", que abarca la mayor parte de su ámbito urbano, razón por la que el estudio y mejora de sus áreas TOD puede tener amplias repercusiones. Igualmente, se constatan como ciudades globales en cuanto a sus dinámicas internacionales, lo que marca la fisonomía de dichas áreas, aunque con matices.

Las ciudades escogidas se encuentran localizadas en los circuitos globales del capital; presentan grandes centros financieros y de negocios a nivel internacional, si bien LA, junto con Londres y París, ocupan los primeros puestos en este ranking mundial. Las tres constituyen ejemplos altamente representativos de una tendencia que se ha hecho creciente para muchas otras grandes ciudades a partir de los ochenta, debido a la globalización (Sassen, 2003; 2013). También Santiago 
y Estambul forman parte de esta red, aunque en menor magnitud (De Mattos, 1999; Turán, 2011), al igual que São Paulo (Mercosur), Ciudad de México, Buenos Aires, Bangkok o Taipei. Con carácter general, el entorno construido de estos nodos densos e hiperconcentrados está formado por un conglomerado de sedes multinacionales, centros especializados, servicios al productor y locales lujosos, desplazando a las tiendas de barrio de carácter más local. Se ubican en las zonas más centrales de las grandes ciudades, buscando su representatividad y conectividad.

LA, considerada como "paradigma de la ciudad tardocapitalista" (García, 2004), "tecnoburbio" (Fishman, 1987) o "posmetrópolis" (Soja, 2001), es pionera en separarse de los modelos urbanos europeos. Su alto nivel de movilidad de población, capital, bienes y servicios se sustenta gracias a su inmensa red de autopistas y carreteras, lo que la convierte en la capital mundial del automóvil, marcando la forma de vida de sus habitantes. Por ello se constata como un modelo de ciudad altamente representativa del crecimiento suburbano disperso de baja densidad, en combinación con grandes centros "posindustriales", con un alto protagonismo del vehículo privado y de sus infraestructuras. Se trata de la "materia prima" que interactúa con sus hubs o concentradores intermodales de transporte, lo que afectará su nivel TOD. Este tipo de urbanización, propia del contexto norteamericano, se puede generalizar a muchas otras áreas metropolitanas de rápido crecimiento del planeta.

Morfológicamente, LA junto con Santiago, parten de un trazado fundacional en malla de origen colonial, si bien el crecimiento de ambas a lo largo del tiempo ha sido bien distinto. Si en la primera, fuera del Downtown, la expansión urbana en general se ha producido mediante sprawl, en la segunda, las transformaciones urbanas más importantes han tenido lugar en su gran centro, en un contexto histórico y cultural diferente que comparte con otras grandes ciudades hispanoamericanas. En Santiago, la modernización de la ciudad tuvo su origen en la evolución de la cuadrícula como orden general, con episodios barrocos y neoclásicos a lo europeo, y en la transformación y densificación de la manzana como unidad predial (Rosas, 1985).

Estambul, aun partiendo como antigua capital de dos imperios y afectada desde los ańos ochenta por políticas económicas neoliberales y proyectos de infraestructura a gran escala, no disfruta de tanta centralidad como las anteriores en la red de urbes globales. Tal condición se ve compensada, sin embargo, por la dinámica económica que supone su localización transfronteriza continental junto al Bósforo. Si bien comparte la misma fenomenología urbana globalizadora de los casos anteriores, su singularidad morfológica, basada en el urbanismo islámico, le confiere otro tipo de representatividad (Turán, 2011). Lo que Haussmann fue a París en el siglo XIX, Henri Prost lo fue a Estambul en los cincuenta, con sus operaciones higienistas mediante el trazado de grandes avenidas a través de tejidos históricos. No obstante, la forma urbana de sus áreas más centrales presenta un trazado irregular, compacto, con escaso espacio libre y manzanas de pequeñas dimensiones, entre las que destacan bazares y mezquitas, lo que afectará de manera particular en la evaluación de sus nodos.

Las cinco constituyen, por tanto, una lista no exhaustiva de ciudades representativas del caso de estudio cuyo perfil, así como los resultados del presente estudio, son extrapolables al de otras muchas ciudades. Los datos de partida provienen 
fundamentalmente de una investigación realizada con medios virtuales convencionales, en la que han participado estudiantes y profesores de grado universitario en Arquitectura. La integridad de los datos se basa en la metodología única empleada para la obtención de los mismos.

\section{Metodología}

La metodología empleada se lleva a cabo mediante el desarrollo de cuatro fases: recopilación de datos, obtención del Índice ToD; análisis multivariable sом y análisis espacial sIG de algunos resultados.

\section{Fase 1. Recopilación de datos}

Los datos de partida se han obtenido a partir de observaciones y mediciones realizadas mediante herramientas convencionales (Google Earth, Google Street View, Wikimapia y cartografía urbana existente a escala del tipo vectorial y raster) y recogidas en formulario. Posteriormente, se les aplica una batería de indicadores, obteniendo los valores de cálculo para el desarrollo de las Fases 2 y 3 (figura 2). Las tablas 1 y 2 muestran los indicadores empleados, los casos de estudio y las puntuaciones asignadas. En la Fase 4 (análisis espacial) los datos de partida se han obtenido a partir de bases de datos oficiales con acceso en abierto.

\section{Fase 2. Obtención del Índice TOD}

Se trata de la valoración o índice global resultante para cada área TOD una vez se suman las puntuaciones parciales obtenidas tras la aplicación de indicadores a los datos de partida. Se usan como base los 21 indicadores recogidos en el formulario TOD Standard versión 2.1 (2014) elaborado por el ITDP de Nueva York (en adelante Norma TOD), agrupados bajo ocho principios (tabla 1). El área TOD queda definida en todos los casos como el entorno construido comprendido en un radio de hasta 450 metros de distancia con centro en cada estación de transporte (véase el principio 4. Transportar, en tabla 1 ).

Existen varios métodos para la evaluación mediante índice del nivel TOD de un área urbana. Para nuestro caso de estudio se ha escogido la Norma TOD, por tratarse de un instrumento eminentemente práctico y accesible para audiencias técnicas no especializadas. Los datos requeridos se pueden recopilar a través de medios convencionales, pudiendo ser objetivamente observados y verificados. Los indicadores y el sistema de puntuación empleados se han diseñado mediante un panel de expertos, y admiten una aplicabilidad genérica e internacional (ITDP, 2014). Las ligeras modificaciones realizadas al formulario original, con objeto de hacer más operativa y ágil la recolección de datos, no suponen una alteración sensible de los resultados. Se debe indicar que cuanto mayor es el valor que alcanza cada indicador, mayor es la puntuación que obtiene, salvo para los indicadores 3.1, 5.1, 8.1, 8.2 y 8.3 en los que esta relación es inversa, debido al tipo de procesamiento de los datos. 
TABLa I | Principios e indicadores empleados, y asignación de puntuación

\begin{tabular}{|c|c|c|c|}
\hline $\begin{array}{l}\text { PRINCIPIOS Y } \\
\text { PUNTUACIÓN } \\
\text { MÁXIMA } \\
\text { S/PRINCIPIO }\end{array}$ & INDICADORES & DESCRIPCIÓN & $\begin{array}{l}\text { PUNTUACIÓN } \\
\text { MÁX. TOTAL: } \\
\text { IOO PTOS. }\end{array}$ \\
\hline \multirow{5}{*}{$\begin{array}{l}\text { 1. Caminar } \\
(\mathrm{CN}): 15 \text { ptos. }\end{array}$} & 1.1 Vías peatonales & $\begin{array}{l}\text { \% de tramos de acera con eliminación de } \\
\text { barreras arquitectónicas }\end{array}$ & 3 ptos. \\
\hline & $\begin{array}{l}1.2 \text { Cruces peato- } \\
\text { nales }\end{array}$ & $\begin{array}{l}\% \text { de cruces entre calles completos y accesibles } \\
\text { en todas direcciones para el peatón }\end{array}$ & 3 ptos. \\
\hline & $\begin{array}{l}1.3 \text { Fachadas visual- } \\
\text { mente activas }\end{array}$ & $\begin{array}{l}\% \text { de longitud de acera en contacto visual con } \\
\text { fachadas activas (en contacto con actividades no } \\
\text { residenciales) }\end{array}$ & 6 ptos. \\
\hline & $\begin{array}{l}\text { 1.4 Fachadas física- } \\
\text { mente permeables }\end{array}$ & $\begin{array}{l}\text { No de accesos peatonales a tiendas y edificios } \\
\text { por cada } 100 \text { metros de fachada existente }\end{array}$ & 2 ptos. \\
\hline & $\begin{array}{l}1.5 \text { Sombra y } \\
\text { refugio }\end{array}$ & $\begin{array}{l}\% \text { de tramos de acera que incorporan elementos } \\
\text { de sombra o refugio }\end{array}$ & 1 pto. \\
\hline \multirow{3}{*}{$\begin{array}{l}\text { 2. Pedalear (PD): } \\
5 \text { ptos. }\end{array}$} & 2.1 Red ciclista & $\begin{array}{l}\text { \% de longitud de calle con carril-bicicletas } \\
\text { seguro y completo (en ambas direcciones, con } \\
\text { pavimento y señalización específicos) }\end{array}$ & 2 ptos. \\
\hline & $\begin{array}{l}2.2 \text { Estacionamiento } \\
\text { para bicicletas en pa- } \\
\text { radas de transporte } \\
\text { público }\end{array}$ & $\begin{array}{l}\text { En la parada de transporte público se } \\
\text { proporcionan instalaciones seguras y diversas } \\
\text { para bicicletas (en parada = } 2 \text { ptos.; en parada } \\
\text { no, pero sí en calles del entorno = } 1 \text { pto.; ni en } \\
\text { parada ni en entorno = } 0 \text { ptos.) }\end{array}$ & 2 ptos. \\
\hline & $\begin{array}{l}\text { 2.3 Estacionamiento } \\
\text { para bicicletas en los } \\
\text { edificios }\end{array}$ & $\begin{array}{l}\text { \% de edificios que proporcionan } \\
\text { estacionamiento seguro para bicicletas en sus } \\
\text { zonas comunes }\end{array}$ & 1 pto. \\
\hline \multirow{2}{*}{$\begin{array}{l}\text { 3. Conectar } \\
\text { (Ст): } 15 \text { ptos. }\end{array}$} & $\begin{array}{l}\text { 3.1 Manzanas } \\
\text { pequeñas }\end{array}$ & Longitud mayor de las manzanas & 10 ptos. \\
\hline & $\begin{array}{l}\text { 3.2 Conectividad } \\
\text { priorizada }\end{array}$ & $\begin{array}{l}\text { Proporción entre el } \mathrm{n}^{\circ} \text { de intersecciones } \\
\text { peatonales y rodadas }\end{array}$ & 5 ptos. \\
\hline $\begin{array}{l}\text { 4. Transportar } \\
\text { (TP): Sí/No }\end{array}$ & $\begin{array}{l}\text { 4.1 Distancia pea- } \\
\text { tonal a la parada de } \\
\text { transporte público } \\
\end{array}$ & $\begin{array}{l}\text { Longitud de la distancia a pie hasta la estación } \\
\text { o parada de transporte público }<450 \mathrm{~m}(5 \\
\text { minutos })\end{array}$ & $\mathrm{s} / \mathrm{n}$ \\
\hline \multirow{3}{*}{$\begin{array}{l}\text { 5. Mezclar (Mz): } \\
15 \text { ptos. }\end{array}$} & $\begin{array}{l}\text { 5.1 Usos } \\
\text { complementarios }\end{array}$ & $\begin{array}{l}\text { Usos residenciales y no-residenciales } \\
\text { combinados dentro de las manzanas o en } \\
\text { manzanas adyacentes }\end{array}$ & 10 ptos. \\
\hline & $\begin{array}{l}\text { 5.2 Accesibilidad a } \\
\text { los alimentos }\end{array}$ & $\begin{array}{l}\% \text { de edificios que se encuentran a } 5 \text { minutos a } \\
\text { pie de una fuente de alimento fresco }\end{array}$ & 1 pto. \\
\hline & $\begin{array}{l}\text { 5.3 Tipologías } \\
\text { residenciales } \\
\text { económicas }\end{array}$ & $\begin{array}{l}\text { \% de tipologías residenciales económicas o } \\
\text { colectivas }\end{array}$ & 4 ptos. \\
\hline $\begin{array}{l}\text { 6. Densificar } \\
\text { (Ds): } 15 \text { ptos. }\end{array}$ & $\begin{array}{l}\text { 6.1 Densidad del } \\
\text { uso del suelo }\end{array}$ & $\begin{array}{l}\text { Densidad media comparada con las condiciones } \\
\text { locales }\end{array}$ & 15 ptos. \\
\hline \multirow{2}{*}{$\begin{array}{l}\text { 7. Compactar } \\
\text { (CP): } 15 \text { ptos. }\end{array}$} & 7.1 Sitio urbano & $\begin{array}{l}\text { No de lados del área de estudio TOD } \\
\text { colindantes con la urbanización existente }\end{array}$ & 10 ptos. \\
\hline & $\begin{array}{l}7.2 \text { Opciones de } \\
\text { transporte }\end{array}$ & $\begin{array}{l}\text { No de diferentes opciones de transporte } \\
\text { accesibles dentro de una distancia caminable }\end{array}$ & 5 ptos. \\
\hline \multirow{3}{*}{$\begin{array}{l}\text { 8. Cambiar } \\
\text { (CB): } 20 \text { ptos }\end{array}$} & $\begin{array}{l}\text { 8.1 Estacionamiento } \\
\text { de coches fuera de la } \\
\text { vía pública }\end{array}$ & $\begin{array}{l}\text { \% de superficie (s/superficie total del área de } \\
\text { estudio dedicada al estacionamiento de vehículos) } \\
\text { fuera de la vía pública (bolsas de aparcamiento) }\end{array}$ & 10 ptos. \\
\hline & $\begin{array}{l}\text { 8.2 Densidad de } \\
\text { accesos rodados }\end{array}$ & $\begin{array}{l}\text { No de accesos para vehículos por cada } \\
100 \text { metros de fachada edificada }\end{array}$ & 2 ptos. \\
\hline & $\begin{array}{l}\text { 8.3 Estacionamiento } \\
\text { en la vía pública y } \\
\text { áreas de circulación }\end{array}$ & $\begin{array}{l}\text { \% de superficie s/totalidad del área de estudio } \\
\text { dedicada a la circulación de vehículos y a su } \\
\text { estacionamiento en la vía pública }\end{array}$ & 8 ptos. \\
\hline
\end{tabular}

FUENTE ELABORACIÓN PROPIA CON BASE EN LA NORMA TOD 


\section{Fase 3. Análisis multivariable som}

Se siguen los pasos definidos por M. S. Silver (2008) para los Sistemas de Apoyo a la Decisión (DSS):

1) Información y funciones de procesado: Los valores de cálculo se obtienen mediante la aplicación de indicadores (véase fase anterior).

2) Conjunto de datos: Los datos recopilados para cada área TOD se han obtenido a partir de observaciones y mediciones convencionales. Coinciden con los datos recopilados en la Fase 1.

3) Modelos: Red neuronal artificial del tipo sом.

El estado del arte demuestra que es muy frecuente el uso de los som como metodología de reducción y clasificación (Hamaina, Leduc \& Moreau, 2012). Comparado con otros métodos de reducción de dimensiones, como el Análisis de Componentes Principales (PCA en inglés) o el Escalado Multidimensional (MDs en inglés), la capacidad de los som de preservar la topología de los datos hace que tenga un uso más eficiente del espacio disponible en la representación del mapa (Skupin \& Agarwal, 2008).

Por otro lado, el som tiene ventajas notables frente a otros métodos de clasificación y conocimiento. Presenta bastante solidez frente a los valores perdidos, aceptando al mismo tiempo datos que muestran una distribución no normal (Zhang, Shi \& Zhang, 2009). Como método de clusterización, el som es más robusto que otros (como, por ejemplo, el K-Means), aunque necesita más tiempo de computación (Bação, Lobo \& Painho, 2005).

El agrupamiento, clusterización o clasificación de los sujetos se realiza mediante un análisis adicional Wardclúster sobre el mapa. Se generan perfiles o prototipos mediante modelado de patrones y tendencias en la información (Weiss \& Indurkhya, 1997). El número final de perfiles se va ajustando dependiendo del objetivo del investigador y de la interpretación de los resultados que se van obteniendo, deteniéndose el proceso cuando la nueva división no aporte conocimiento extra.

Para cumplir con las recomendaciones de la American Statistical Association (Wasserstein \& Lazar, 2016) para cada variable y perfil, además de la significación estadística mediante la Prueba T-Student bilateral (pvalor $\leq 0.05$ ), se calcula su tamaño a partir del efecto. Este se entiende como el cociente de la diferencia de la media entre el grupo experimental (perfil) y la media del grupo control (media de la población), dividido entre la desviación estándar de la población (Coe \& Merino, 2003).

En la figura 2 se muestran los resultados de la clasificación en seis perfiles. Igualmente, se indica el Tamańo del Efecto (Es) para cada atributo o variable que interviene en la construcción del perfil: $(+++)$ ES positivo alto $(++)$, ES positivo medio $(+)$, ES positivo bajo $(-)$, ES negativo bajo $(--)$, ES negativo medio, y (- - ) ES negativo alto (Cohen, 1988), obteniéndose una información relevante del efecto que tiene cada una de las variables en la definición y singularidad de cada perfil. El es consiste en la magnitud del "efecto" que 
ejerce en cada caso de estudio la pertenencia a un determinado perfil, sobre los valores alcanzados en cada variable. Constituye, por tanto, una medida de la diferenciación de cada perfil en relación con la totalidad de los casos de estudio.

4) Representaciones visuales: Se obtiene una representación bidimensional de las instancias de partida, en la que cada una de ellas tiene por "vecina" la instancia con cualidades más semejantes. En la misma cartografía se representan las agrupaciones de las instancias en los distintos perfiles conformados. Esta representación se suele completar con un mapa (monovariable) por cada una de las variables que construyen el mapa sом (figura 1).

En la interpretación de los resultados hay que tener en cuenta que la combinación del conocimiento experto con los resultados som requiere cierta creatividad (Kauko, 2005), ya que no son en absoluto inmediatos ni obvios.

\section{Fase 4. Análisis espacial sig de algunos resultados}

La representación espacial mediante SIG de los datos obtenidos para cada área TOD va a permitir completar la información derivada de los análisis cuantitativos y estadísticos practicados, lo que ayuda a la comprensión de la realidad urbana de estos entornos. Como ejemplo de ello se han analizado las áreas Saint-Nazare, Vaugirard y Opera. Se trata de áreas que, aun perteneciendo a la misma ciudad y presentando un Índice TOD similar, emergen con una significación estadística distinta.

Para el análisis señalado se ha usado el software ArcMap. Los principales comandos utilizados han sido: "Create Buffers", "Summarize Within" y "Connect Origin to Destination (Travel Mode: Walking Time)". Los datos de partida se han descargado de las siguientes bases de datos geolocalizados en abierto: Open Data Paris (servicios de la ciudad de París), Open Data Apur (agencia de urbanismo parisién) y Open Data Ratp (agencia de transportes parisién). Los datos sobre viales se han obtenido de la Direction de la Voirie et des Déplacements - Division des plans de voirie de la Ville de Paris. La localización de los proyectos de carácter público se ha obtenido del Plan Local de Urbanismo (plu) de París. Cuando los datos necesarios no existían en las bases mencionadas, se obtuvieron mediante observaciones convencionales a través de Google Street View.

Solo se representan gráficamente las variables que han alcanzado valores claramente divergentes en los distintos casos, situándolos en perfiles distintos. Se trata de los valores que alcanzan los indicadores $1.5,3.1$ y 3.2 para la comparativa Opera Saint-Lazare, y 1.3, 2.1, 3.1 y 7.2 para Vaugirard - Saint-Lazare (véase figuras 3 y 4).

En el análisis de la métrica 1.3 también se consideraron activas las fachadas de los espacios ajardinados vallados pero visualmente permeables. Las fachadas edificadas con vistas a los espacios ferroviarios (espacios abiertos no accesibles al público) se han considerado inactivas. Para la métrica 1.5, el área de sombra alrededor de los árboles se calculó mediante un buffer de 6,60 $\mathrm{m}$ de radio alrededor del punto de ubicación, que es el radio medio de los árboles más comunes de la zona (Chanes, 2009). Finalmente, en el caso del indicador 3.2, las galerías cubiertas y los caminos principales de los parques públicos se han considerado como vías peatonales en las intersecciones. 


\section{Resultados}

Una vez procesados los datos de partida, se obtienen los Índices ToD para cada área (tabla 2), así como seis perfiles o clústeres (figuras 1 y 2) a partir del análisis som. En este análisis no entran en juego las puntuaciones del Índice тоD de cada área, sino únicamente las variables que las generan. Finalmente, se dan a conocer los resultados del análisis espacial sig practicado.

TABLA 2 Índice TOD. Puntuaciones parciales y globales para cada área según principios de la Norma TOD, agrupadas por ciudad y ordenadas según total

\begin{tabular}{|c|c|c|c|c|c|c|c|c|c|c|}
\hline \multicolumn{2}{|c|}{ PRINCIPIOS NORMA TOD } & $\mathrm{CN}$ & PD & $\mathrm{CT}$ & TP & MZ & DS & $\mathrm{CP}$ & $\mathrm{CB}$ & TOTAL \\
\hline \multicolumn{2}{|c|}{ VALOR MÍN.-MÁX. } & $0-15$ & $0-5$ & $0-15$ & sí/NO & O-I 5 & $0-15$ & O-I 5 & $0-20$ & $0-100$ \\
\hline \multicolumn{2}{|l|}{ MEDIA } & 7,29 & $\mathbf{I , 2 3}$ & 7,77 & - & 12,39 & 6,97 & 13,60 & I0,30 & 59,48 \\
\hline \multicolumn{2}{|l|}{ MEDIANA } & 7 & $\mathbf{I}$ & 7 & - & 14 & 7 & 15 & IO & 62 \\
\hline \multicolumn{2}{|l|}{ MODA } & 7 & $\mathbf{I}$ & 7 & - & 15 & 7 & 15 & 7 & 65 \\
\hline CIUDAD & ÁREA TOD & \multicolumn{9}{|c|}{ VALORES POR ÁREA TOD } \\
\hline \multirow{8}{*}{ Londres } & Oxford Circus & 12 & 1 & 13 & sí & 14 & 15 & 15 & 10 & 80 \\
\hline & Picadilly Circus & 10 & 3 & 11 & sí & 15 & 15 & 15 & 7 & 76 \\
\hline & Aldgate & 8 & 1 & 13 & sí & 13 & 7 & 15 & 12 & 69 \\
\hline & Regent Street & 9 & 1 & 9 & sí & 14 & 7 & 8 & 17 & 65 \\
\hline & Marylebone Station & 7 & 1 & 11 & sí & 14 & 0 & 11 & 20 & 64 \\
\hline & Waterloo & 5 & 3 & 7 & sí & 15 & 7 & 15 & 10 & 62 \\
\hline & Euston & 4 & 1 & 5 & sí & 14 & 0 & 15 & 15 & 54 \\
\hline & London Bridge & 1 & 1 & 7 & sí & 14 & 0 & 15 & 15 & 53 \\
\hline \multirow{6}{*}{ París } & Place de Republique & 8 & 3 & 5 & sí & 15 & 15 & 15 & 17 & 78 \\
\hline & Olympiades & 7 & 2 & 9 & sí & 15 & 15 & 15 & 12 & 75 \\
\hline & Gare de l'est & 12 & 1 & 7 & sí & 15 & 15 & 15 & 7 & 72 \\
\hline & Vaugirard & 8 & 1 & 13 & sí & 15 & 7 & 13 & 10 & 67 \\
\hline & Opera & 9 & 1 & 11 & sí & 14 & 0 & 15 & 17 & 67 \\
\hline & Gare Saint Lazare & 13 & 2 & 3 & sí & 15 & 7 & 15 & 7 & 62 \\
\hline \multirow{5}{*}{ LA } & Metro Center & 7 & 2 & 2 & sí & 6 & 15 & 13 & 12 & 57 \\
\hline & Pershing Square & 8 & 0 & 3 & sí & 10 & 7 & 14 & 5 & 47 \\
\hline & Westlake-McA. Park & 7 & 0 & 7 & sí & 8 & 0 & 11 & 7 & 40 \\
\hline & Wilshire Blvd-Park & 5 & 2 & 1 & sí & 9 & 7 & 15 & 0 & 39 \\
\hline & Soto Station & 1 & 2 & 7 & sí & 2 & 0 & 15 & 5 & 32 \\
\hline \multirow{7}{*}{$\begin{array}{l}\text { Santiago de } \\
\text { Chile }\end{array}$} & Plaza de Armas & 10 & 3 & 6 & sí & 15 & 7 & 15 & 15 & 71 \\
\hline & Universidad de Chile & 10 & 2 & 7 & sí & 13 & 7 & 15 & 12 & 66 \\
\hline & Universidad Católica & 7 & 0 & 9 & sí & 15 & 7 & 11 & 7 & 56 \\
\hline & Plaza de Ciudadanía & 7 & 1 & 9 & sí & 10 & 7 & 15 & 5 & 54 \\
\hline & Baquedano & 14 & 1 & 7 & sí & 10 & 0 & 9 & 8 & 49 \\
\hline & Manuel Montt & 2 & 1 & 5 & sí & 10 & 7 & 15 & 5 & 45 \\
\hline & Plaza Egańa & 1 & 1 & 3 & sí & 9 & 7 & 15 & 5 & 41 \\
\hline \multirow{5}{*}{ Estambul } & Laleli-Üniversite & 9 & 0 & 11 & sí & 10 & 7 & 13 & 15 & 65 \\
\hline & Aksaray & 6 & 0 & 11 & sí & 15 & 7 & 11 & 15 & 65 \\
\hline & Findikzade & 6 & 0 & 11 & sí & 15 & 7 & 13 & 12 & 64 \\
\hline & Sishane & 7 & 1 & 9 & sí & 15 & 7 & 9 & 7 & 55 \\
\hline & Plaza Taksim & 6 & 0 & 9 & sí & 10 & 7 & 15 & 7 & 54 \\
\hline
\end{tabular}




\section{Mapa de Perfiles ToD}

Cada uno de los perfiles contiene varias localizaciones o áreas TOD, excepto la de Opera (París), que por su singularidad conforma un perfil ella sola. Cada área recibe su nombre a partir del nodo de transporte en torno al cual se desarrolla. La posición de cada área TOD en el mapa som tiene que ver con su comportamiento iterativo en el análisis multivariable. De esta manera, las áreas que tienen un comportamiento semejante figuran próximas unas de otras, constituyendo un perfil o clúster. Por el contrario, las localizaciones TOD que presentan un comportamiento diferente se muestran alejadas entre sí en el mapa, pudiendo formar parte de perfiles distintos.

FIgURA I | Extracto del conjunto de mapas som monovariables para la clasificación de seis perfiles
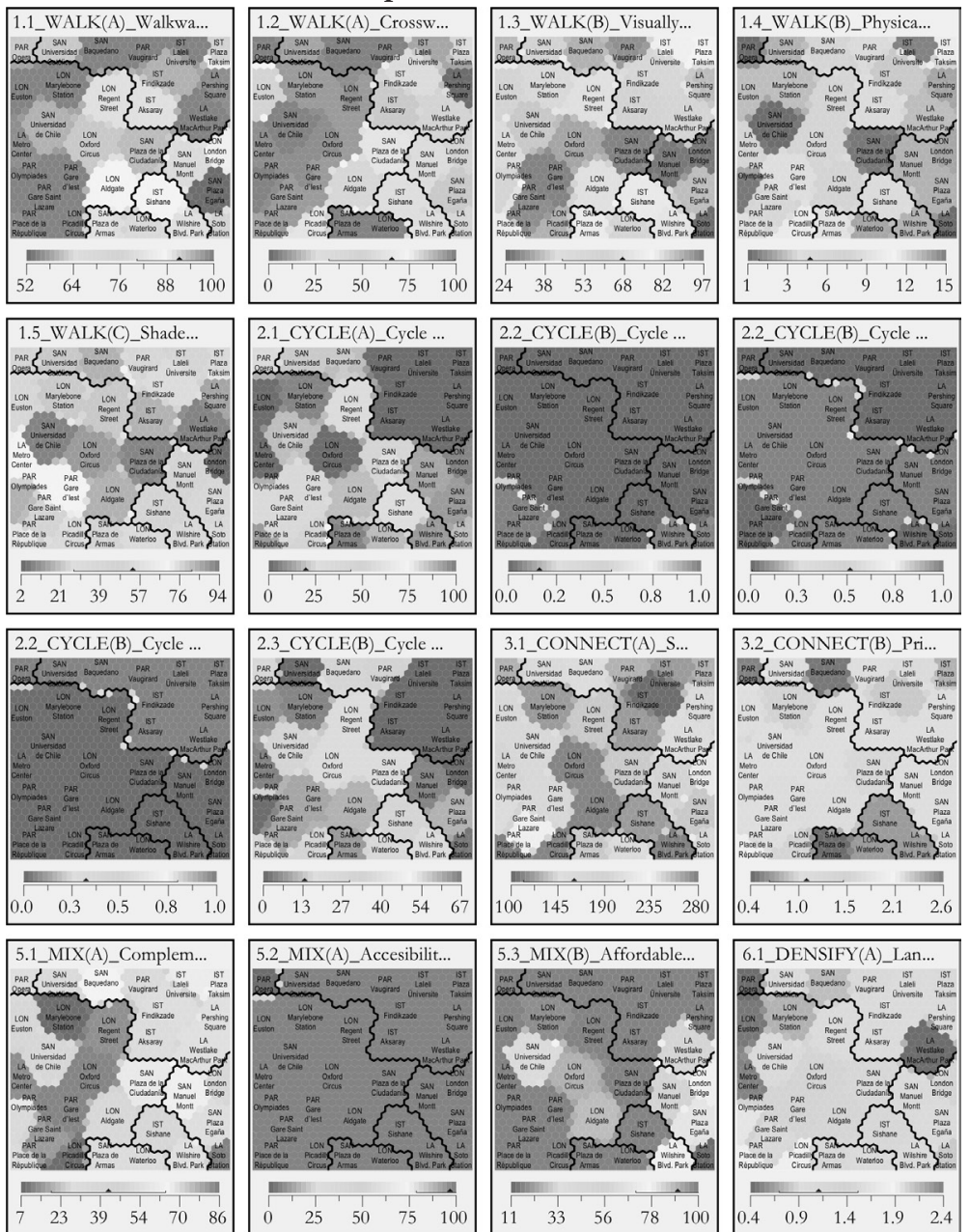

NOTA

EN CADA UNO DE LOS MAPAS SE MUESTRAN LOS RESULTADOS DEL ANÁLISIS PARA CADA UNA DE LAS VARIABLES (INDICADORES) DE FORMA INDEPENDIENTE. LA AGRUPACIÓN DE LAS INSTANCIAS O MAPA SOM ES EL RESULTADO DE LA COMPOSICIÓN CONJUNTA DE LAS REPRESENTACIONES MONOVARIABles (VÉASE PUNTO 4 DE LA FASE 3 DEL APARTAdo METOdológico).

FUENTE ELABORACIÓN PROPIA 


\section{Caracterización de los perfiles ToD}

Se obtienen seis perfiles del modelo de clasificación calculado. Se ha omitido la tabla con los valores obtenidos en el agrupamiento de los perfiles som (confianza o p-valor obtenido en la prueba T-Student bilateral y otra información estadística) con objeto de facilitar la lectura de los resultados. En la figura 2 se recogen los valores del Tamańo del Efecto (Es) mediante signos (positivos o negativos) y la significación estadística (recuadro enmarcado y sombreado si es significativo) que ejerce la pertenencia al perfil en los valores que alcanza cada variable o indicador.

Como aclaración, por ejemplo, un Es positivo alto $(+++)$ y significativo estadísticamente determina que los valores del indicador en cuestión son elevados para prácticamente la totalidad de las áreas ToD integradas en el perfil correspondiente. Si no es estadísticamente significativo, solo presentará un valor elevado para algunas de ellas.

Figura 2 Perfiles SOM, con indicación del Índice Tod de cada área

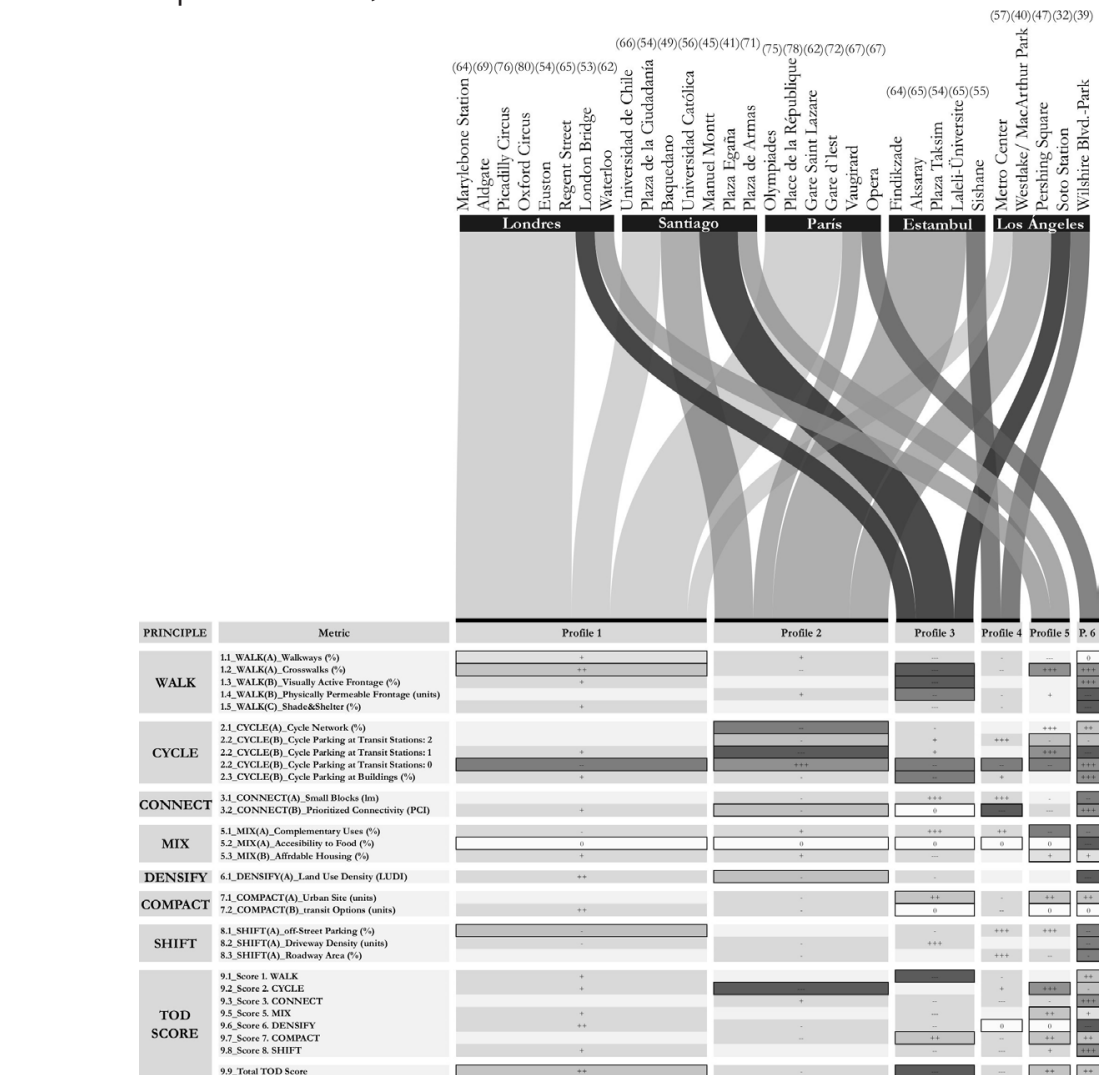

CUANTO MAYOR ES El NÚMERo De Signos positivos o Negativos (Es) QUe PResenta CADA INDICADOR (FILAS) EN CADA PERFIL (COLUMNAS), MAYOR SERÁ SU INFLUENCIA EN LA CONFIGURACIÓN DEL MISMO. ESTA INFLUENCIA PODRÁ SER EJERCIDA POR TODAS LAS INSTANCIAS DEL PERFIL (CON SIGNIFICACIÓN ESTADÍSTICA) O SOLO POR ALGUNAS DE ELLAS (SIN SIGNIFICACIÓN). LOS RESULTADOS TOTALES DE CADA PRINCIPIO SON MOSTRADOS (TOD SCORE), PERO NO FORMAN PARTE DEL PROCESO ITERATIVO DE CÁLCULO DE LA RED NEURONAL PARA EVITAR DISTORSIONES. ADICIONALMENTE, JUNTO A CADA NODO SE MUESTRA SU ÍNDICE TOD (SEGÚN FASE 2 ).

FUENTE ELABORACIÓN PROPIA 


\section{Perfil 1: Entornos densos, intermodales y seguros para el peatón}

Se trata de un perfil caracterizado por presentar: bastantes cruces peatonales completos y accesibles en todas las direcciones, un Índice TOD elevado, algunos tramos de acera sin barreras arquitectónicas, y estacionamiento para bicicletas en las paradas de transporte público.

Solo algunas áreas ToD de este grupo presentan: densidad del uso del suelo elevada con respecto a la media de la zona, estaciones de transporte con un buen nivel de intermodalidad y un puntaje alto en el principio de densificar. El resto de las variables evaluadas destacan ligeramente (ES positivo bajo o medio), con respecto a la media de la globalidad de los casos de estudio.

Es el perfil que más áreas TOD engloba (42\%), con una clara representación del caso europeo (71\% del total de las áreas europeas). Comprende áreas ToD de Londres (76\%), Santiago (29\%), París (66\%) y LA (20\%). Se trata de un perfil mayormente positivo pero relativamente medio, pues los valores de la mayoría de sus variables son cercanos a la media global, lo que es usual cuando la muestra comprende bastantes ejemplares. Sin embargo, contiene las áreas de Índice TOD más elevado, salvo excepciones, con valores que oscilan entre 80 y 54 puntos.

Las áreas TOD del perfil presentan, con significación estadística: ES positivo medio para los indicadores 1.2 (véase tabla 1) y 9.9 (Total TOD); ES positivo bajo para el indicador 1.1; Es negativo medio para el indicador 2.2 (=0 ptos.). Sin significación estadística: es positivo medio para los indicadores 6.1, 7.2 y 9.6 (Densify).

\section{Perfil 2: Entornos permeables pero poco densos, e inseguros para ciclistas}

Se caracteriza por presentar: paradas de transporte público y calles sin apenas estacionamiento para bicicletas; en general no existen calles con carriles-bicicletas seguros y completos; una puntuación muy baja en el principio de pedalear; algunos cruces rodados sin aceras y una densidad de uso del suelo algo baja con respecto a la media de la zona.

Algunas áreas TOD de este grupo presentan: numerosos cruces peatonales incompletos y poco accesibles, y una puntuación baja en el principio de compactar.

Se ocupa de un $29 \%$ del total de áreas examinadas, con una clara representación del caso euro-asiático. Comprende áreas ToD de Santiago (29\%), París (17\%), Estambul (80\%) y LA (40\%). Estas áreas presentan un Índice TOD que oscila entre 67 y 40 puntos.

El perfil muestra, con significación estadística: es positivo alto para el indicador 2.2 (=0 ptos.). ES negativo alto para los indicadores 2.2 (=1 pto.) y 9.2 (Cycle). ES negativo medio para el indicador 2.1. Es negativo bajo para los indicadores $2.2(=2$ ptos.), 3.2 y 6.1. Sin significación estadística: es negativo medio para los indicadores 1.2 y 9.7 (Compact).

Perfil 3: Entornos compactos pero monótonos, poco permeables e inseguros para el peatón

Este perfil presenta: las áreas TOD rodeadas de zonas urbanizadas, buena puntuación en el principio de compactar, cruces entre calles incompletos y poco accesibles, aceras sin apenas contacto visual con fachadas activas, muy baja puntuación en el 
principio de caminar, un Índice TOD muy bajo, un número escaso de accesos peatonales a tiendas y edificios a lo largo de las fachadas, sin apenas estacionamiento seguro y diverso para bicicletas en los edificios, aunque existentes para algunas áreas en las paradas de transporte público y en calles de alrededor, y una proporción entre cruces peatonales y rodados en la media, al igual que ocurre con el nivel de intermodalidad.

Algunas áreas TOD presentan: manzanas grandes, plantas bajas predominantemente residenciales, aceras frecuentemente interrumpidas por accesos rodados a los edificios, aceras con barreras arquitectónicas y sin sombra o refugio, presencia de tipologías unifamiliares, una puntuación pésima en el principio de mezclar, y baja en los principios de conectar, densificar y cambiar.

Comprende un 13\% del total de áreas ToD examinadas. Engloba áreas de Londres (12\%), Santiago (29\%) y LA (20\%). Presentan un Índice ToD que oscila entre 53 y 32 ptos. Se trata del perfil que contiene los Índices ToD de valoración más baja.

Muestra, con significación estadística: es positivo medio para los indicadores 7.1 y 9.7 (Compact). Es negativo alto para los indicadores 1.2, 1.3, 9.1 (Walk) y 9.9 (Total TOD). Es negativo medio para los indicadores $1.4,2.2$ (=0 ptos.) y 2.3 . Los valores obtenidos para los indicadores 3.2 y 7.2 se encuentran en la media. Sin significación estadística: Es positivo alto para los indicadores 3.1, 5.1 y 8.2. ES negativo alto para los indicadores $1.1,1.5,5.3$ y 9.5 (Mix). Es negativo medio para los indicadores 9.3 (Connect), 9.6 (Densify) y 9.8 (Shift).

Perfil 4: Entornos poco compactos, poco seguros y con un alto protagonismo del vehículo privado

Presenta: un elevado número de cruces rodados sin aceras y bastantes paradas de transporte público con estacionamiento para bicicletas.

Algunas áreas TOD de este perfil muestran: manzanas grandes, numerosas bolsas de aparcamiento en superficie, bastante superficie destinada a la circulación de vehículos y a su aparcamiento en vía pública, un puntaje muy bajo en los principios de conectar y cambiar, así como en la valoración o Índice TOD general. Pocos cruces peatonales completos y accesibles en todas las direcciones, baja intermodalidad y baja valoración en el principio de compactar.

Se ocupa de un $6 \%$ del total de áreas examinadas. Comprende áreas TOD de Estambul (20\%) y de LA (20\%). Muestran un Índice TOD que oscila entre 55 y 39 ptos.

Este perfil presenta, con significación estadística: ES negativo alto para el indicador 3.2. Es negativo medio para el indicador 2.2 (=0 ptos.). Sin significación estadística: ES positivo alto para los indicadores 2.2 (=2 ptos.), 3.1, 8.1 y 8.3. ES negativo alto para los indicadores 9.3 (Connect), 9.8 (Shift) y 9.9 (Total TOD). Es negativo medio para los indicadores $1.2,7.2$ y 9.7 (Compact).

\section{Perfil 5: Entornos compactos, mixtos y seguros, aunque incómodos}

Perfil caracterizado por presentar: cruces peatonales completos y accesibles en todas las direcciones, estacionamiento para bicicletas en calles en torno a la parada de transporte público, bastantes zonas urbanizadas en torno al área de estudio, usos 
mixtos en las plantas bajas, una puntuación muy alta en el principio de pedalear y alta en los principios de mezclar, compactar y en la valoración ToD global.

Algunas áreas TOD de este perfil presentan: un número elevado de calles con carriles-bicicletas seguros y completos, bastantes bolsas de aparcamiento en superficie fuera de la vía pública, numerosas calles con barreras arquitectónicas, muchos cruces rodados sin aceras y escasa superficie destinada a la circulación de vehículos y a su estacionamiento en la vía pública.

Comprende el 12\% de las áreas TOD de Londres y el 14\% de las de Santiago, constituyendo un $6 \%$ del total de las áreas examinadas. Se trata del perfil mejor valorado (ES positivo alto/medio en muchas de sus variables estadísticamente significativas, pocos ES negativos). Las áreas que contiene presentan un Índice TOD que oscila entre 71 y 62 ptos., puntuación elevada con respecto a la media global.

Presenta, con significación estadística: Es positivo alto para los indicadores 1.2, 2.2 (=1 pto.) y 9.2 (Cycle). Es positivo medio para los indicadores 7.1, 9.5 (Mix), 9.7 (Compact) y 9.9 (Total TOD). Es negativo medio para los indicadores 2.2 (=0 ptos.) y 5.1. Sin significación estadística: Es positivo alto para los indicadores 2.1 y 8.1. ES negativo alto para los indicadores 1.1 y 3.2. es negativo medio para el indicador 8.3.

\section{Perfil 6: Entornos compactos, vibrantes y seguros, aunque poco densos}

Presenta: cruces completos y accesibles en todas direcciones, alto contacto visual con las actividades no residenciales de las plantas bajas, paradas de transporte sin estacionamiento para bicicletas, que sí se localizan en el interior de los edificios; puntuación muy alta en los principios de conectar y cambiar. Numerosas calles con carriles-bicicletas seguros y completos, bastantes zonas urbanizadas en torno al área de estudio, puntuación alta en los principios de caminar, compactar y en la valoración toD global. Muy pocos accesos peatonales a locales y edificios, aceras sin elementos de sombra y refugio, calles sin estacionamiento para bicicletas ni fuentes de alimento fresco, densidad por debajo de la media de la zona, con un valor muy bajo en el principio de densificar. Manzanas pequeñas, usos mixtos en las plantas bajas, pocas bolsas de aparcamiento en superficie y aceras sin apenas interrupciones por accesos rodados a los edificios.

Comprende una sola área Tod (3\% del total). Se trata del perfil más extremo (muchas variables con Es positivos altos y con es negativos altos) y menos numeroso en cuanto a casos de estudio se refiere, con un solo ejemplar debido a su singularidad. Por esta última razón, todos los casos son significativos. El área de este perfil presenta un Índice TOD de 67 puntos.

Presenta, con significación estadística: Es positivo alto en los indicadores: 1.2 , 1.3, 2.2 (=2 ptos.), 2.3, 9.3 (Connect) y 9.8 (Shift). Es positivo medio en: 2.1, 7.1, 9.1 (Walk), 9.7 (Compact) y 9.9 (Total Tod). Es negativo alto en: 1.4, 1.5, 2.2 (=1 pto.), 5.2, 6.1 y 9.6 (Densify). Es negativo medio en: 3.1, 5.1, 8.1 y 8.2. 


\section{Representación espacial}

A continuación se lleva a cabo un ejemplo de análisis espacial mediante sIG, con objeto de validar y pormenorizar los análisis cuantitativos mostrados en los resultados anteriores. Para ello se analizan tres de los casos de estudio: Vaugirard, Gare Saint-Lazare y Opera, según las condiciones establecidas en el apartado metodológico. Los resultados de este estudio, que serán discutidos en los apartados correspondientes, ponen de relevancia la importancia del análisis espacial como herramienta gráfica complementaria a otros métodos.

\section{TABLA 3 $\mid$ Tabla resumen de resultados del análisis SIG}

Vaugirard:

\begin{tabular}{|l|l|l|l|c|}
\hline \multicolumn{1}{|c|}{ PRINCIPIO } & \multicolumn{1}{|c|}{ VARIABLES } & \multicolumn{1}{c|}{ MEDICIÓN } & \multicolumn{1}{c|}{ NORMA TOD } & VALOR \\
\hline 1. Caminar & 1.3 Fachadas visualmente activas & $89,4 \%$ & $\geq 80 \%$ & 5 \\
\hline 2. Pedalear & 2.1 Red ciclista & $>800 \mathrm{~m}$ & $<50 \%$ longitud calles & 0 \\
\hline 3. Conectar & 3.1 Manzanas pequeñas & $13,3 \%>\mathrm{L}=190 \mathrm{~m}$ & Más del $10 \%>\mathrm{L}=190 \mathrm{~m}$ & 0 \\
\hline 7. Compactar & 7.2 Intermodalidad & 4 modos* & $\geq 4$ modos transporte & 5 \\
\hline
\end{tabular}

* i línea de metro de alta capacidad (metro i 2), 8 líneas de bus regulares (bus 39, 62, 70, 80, 88, 89, 91, 95), I LÍNEA CICLISTA COMPARTIDA, ACCESO PEATONAL

Gare Saint-Lazare:

\begin{tabular}{|l|l|l|l|c|}
\hline \multicolumn{1}{|c|}{ PRINCIPIO } & \multicolumn{1}{|c|}{ VARIABles } & \multicolumn{1}{c|}{ MEDICIÓN } & \multicolumn{1}{c|}{ NORMA TOD } & VALOR \\
\hline \multirow{2}{*}{ 1. Caminar } & 1.3 Fachadas visualmente activas & $92,2 \%$ & $\geq 90 \%$ & 6 \\
\cline { 2 - 5 } & 1.5 Sombra y refugio & $17,40 \%$ & $<75 \%$ & 0 \\
\hline \multirow{2}{*}{ 2. Pedalear } & 2.1 Red ciclista & $>3000 \mathrm{~m}$ & $<50 \%$ longitud calles & 0 \\
\hline \multirow{2}{*}{ 3. Conectar } & 3.1 Manzanas pequeñas & $25,4 \%>\mathrm{L}=190 \mathrm{~m}$ & Más del $10 \%>\mathrm{L}=190 \mathrm{~m}$ & 0 \\
\cline { 2 - 5 } & 3.2 Conectividad priorizada & 0,98 & $0,5 \leq \mathrm{ICP}<1$ & 1 \\
\hline 7. Compactar & 7.2 Intermodalidad & 5 modos* & $\geq 4$ modos transporte & 5 \\
\hline
\end{tabular}

Opera:

\begin{tabular}{|l|l|l|l|c|}
\hline \multicolumn{1}{|c|}{ PRINCIPIO } & \multicolumn{1}{|c|}{ VARIABLES } & \multicolumn{1}{c|}{ MEDICIÓN } & \multicolumn{1}{c|}{ NORMA TOD } & VALOR \\
\hline 1. Caminar & 1.5 Sombra y refugio & $22 \%$ & $<75 \%$ & 0 \\
\hline \multirow{2}{*}{ 3. Conectar } & 3.1 Manzanas pequeñas & $28,09 \%>\mathrm{L}=190 \mathrm{~m}$ & Más del $10 \%>\mathrm{L}=190 \mathrm{~m}$ & 0 \\
\cline { 2 - 5 } & 3.2 Conectividad priorizada & 0,89 & $0,5 \leq \mathrm{ICP}<1$ & 1 \\
\hline 7. Compactar & 7.2 Intermodalidad & 5 modos* & $\geq 4$ modos transporte & 5 \\
\hline
\end{tabular}

FUENTE ELABORACIÓN PROPIA 
FIgURA 3 | Análisis sig áreas Gare Saint-Lazare (izq.) - Opera (der.). Indicadores $1.5,3.1$ y 3.2
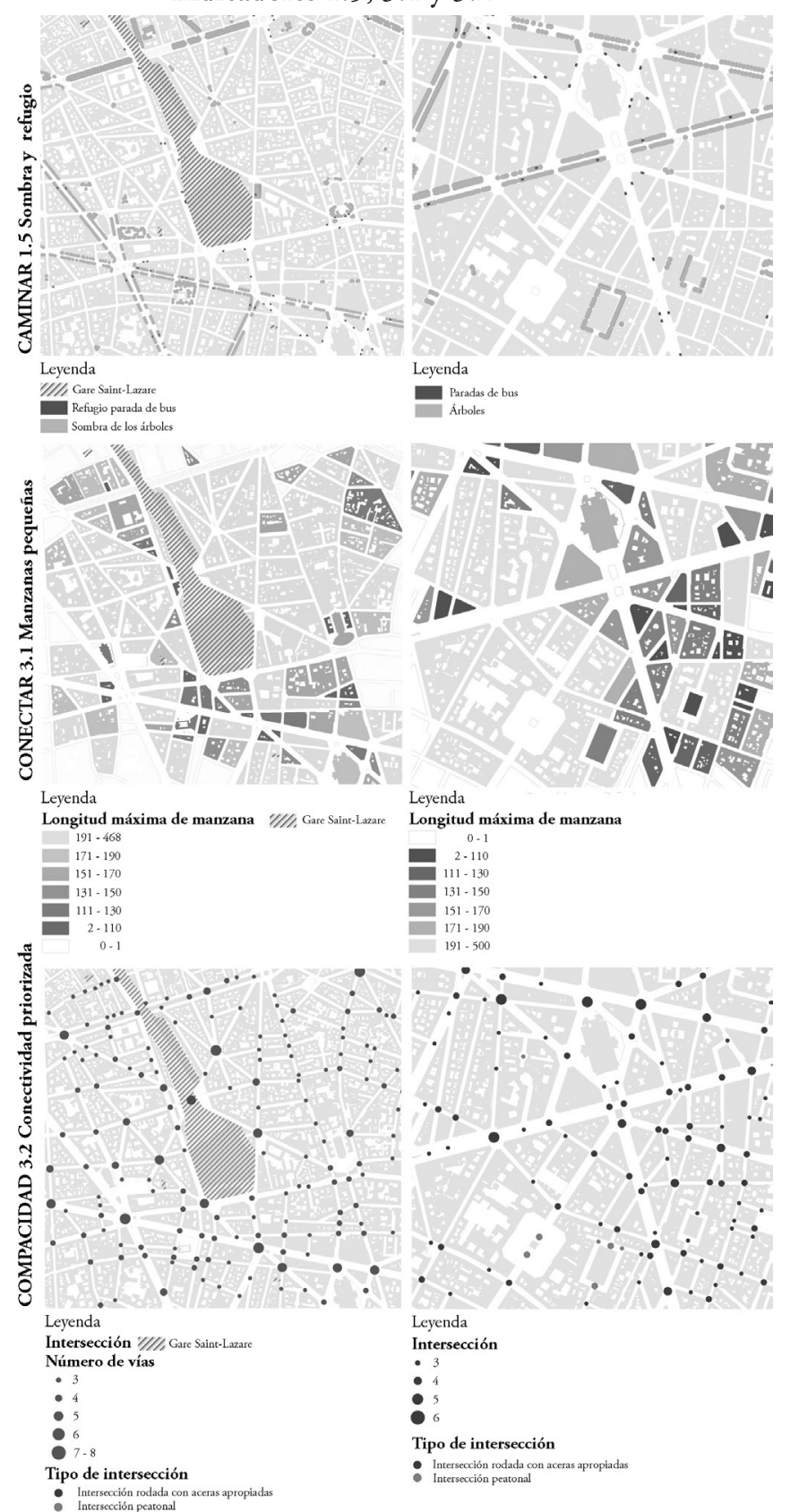

nota EN LAS Figuras SE MUESTRAN, PARA CADA CASO DE ESTUdio: LA SOMBRA y REFUgio QUE PROPORCIONAN LOS APEADEROS DE TRANSPORTE Y LOS ÁRBOLES (INDICADOR I.5); LA LONGITUd MÁXIMA DE LAS MANZANAS, SEGÚN COLOR (3.I); Y EL NÚMERO DE CALLES EN CADA CRUCE, DISTINGUIENDO ENTRE INTERSECCIONES RODADAS Y PEATONALES (3.2).

FUENTE ELABORACIÓN PROPIA 
FIGURA 4 | Cartografía sig áreas Gare Saint-Lazare (izq) y Vaugirard (der.). Indic. 1.3, 2.1, 3.1 y 7.2

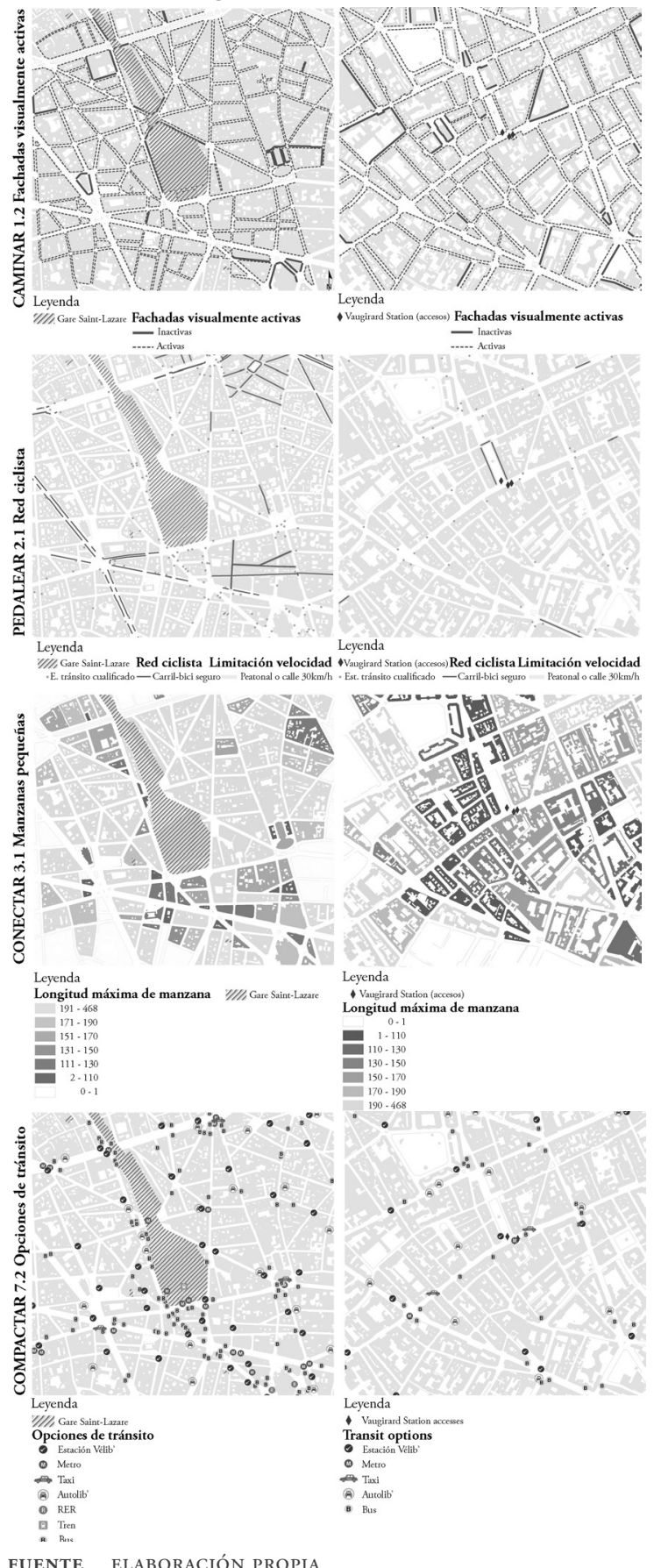

NOTA

EN LAS FIGURAS SE MUESTRAN, PARA CADA CASO DE ESTUDIO: LA LONGITUD DE LAS FACHADAS EN CONTACTO VISUAL, Y SIN ÉL, CON ACTIVIDADES NO RESIDENCIALES EN PLANTA BAJA (INDICADOR I.3); LOS CARRILESBICICLETAS SEGUROS Y LAS CALLES PEATONALES O LENTAS, INDICANDO LOS APARCAMIENTOS PARA BICICLETAS (MEDIANTE PUNTOS) (2.I); LA LONGITUD MÁXIMA DE LAS MANZANAS, SEGÚN COLOR (3.I); Y EL NÚMERO DE MOdOS DE TRANSPORTE DISPONIBLES, SIENDO: RER=TRENES DE CERCANÍAS SUBURBANOS. VÉLIB'=SISTEMA DE BICICLETAS COMPARTIDAS A GRAN ESCALA (INAUGURADO EN PARÍS EN 2007). AUTOLIB' $=$ SERVICIO DE COCHE ELÉCTRICO COMPARTIDO (INAUGURADO EN PARÍS EN 2OII) (INDICADOR 7.2). 


\section{Discusión}

\section{Índices iguales vs. perfiles distintos}

A la vista de los resultados para cada método de evaluación, se ha de destacar la obtención de perfiles som que comprenden áreas con un Índice TOD muy desigual. También se observa justo lo contrario: áreas que presentando un índice muy similar figuran en perfiles diferentes. Esta apreciación, más que contradictoria, ha de entenderse como complementaria desde la particularidad de cada método. El Índice TOD proporciona una valoración global desde la asignación de una puntuación a partir de los valores que adquiere cada variable en cada caso. En cambio, el análisis som atiende a un comportamiento global de las variables en cada caso; o dicho de otra manera, al peso o la coincidencia y amplitud de la variabilidad de cada una de las variables para con las demás en cada caso, lo que permite asociaciones más avanzadas y pormenorizadas. Esto supone una información extra sobre la mera adición de los valores parciales que maneja el Índice ToD.

De esta manera observamos, por ejemplo, cómo todas las áreas TOD que conforman el Perfil 2 tienen en común un comportamiento estadísticamente significativo con valores negativos para casi todas las variables relacionadas con el principio de pedalear. Esta coincidencia determina su agrupación y el número de áreas que la conforman. Podríamos decir que, por encima de cualquier otro factor, se trataría de un perfil claramente caracterizado por las particularidades de su red ciclista, que se encuentran por debajo de la media global.

En este mismo perfil vemos que comprende fundamentalmente áreas pertenecientes a Estambul, LA y Santiago: i) áreas propias de una cultura sin demasiada tradición ciclista, con carencias en cuanto a las condiciones de la infraestructura no motorizada se refiere, en el caso de Estambul; ii) de gran extensión, baja densidad y alto predominio del automóvil como medio de desplazamiento, en el caso de LA; y iii) de áreas que presentan una escala urbana ajena a la bicicleta, coincidentes con arterias y nudos de infraestructuras rodadas de gran capacidad, en el caso de Santiago (figura 5).

\section{FIGURA 5 | Westlake/McArthur Park, LA (izq.) y Baquedano, Santiago (der.)}
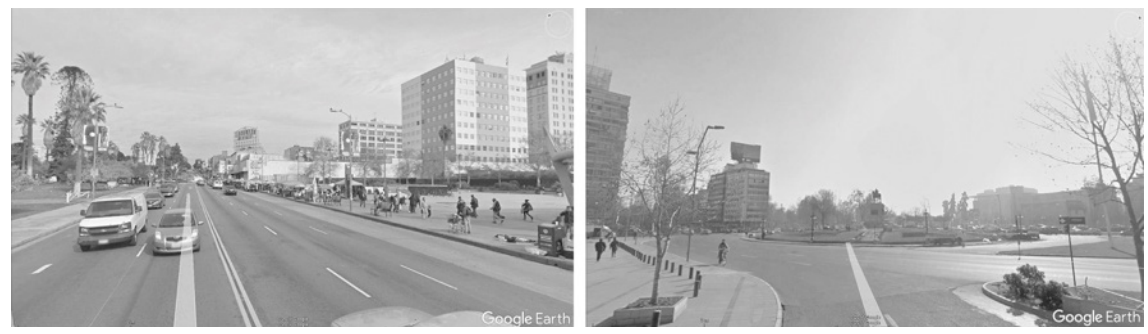

FUENTE GOOGLE STREET VIEW (20I7)

El caso del Perfil 3 es similar al anterior, pero relativo a "caminar". Todas las variables relacionadas con este principio presentan valores negativos elevados y casi todas con significación estadística. Por lo tanto, el peso de las variables que comprenden 
el principio de caminar es determinante en la configuración de este perfil. Este efecto negativo se ve reforzado por la existencia de algunas áreas provistas de grandes manzanas, lo que impide que las rutas peatonales sean cortas, directas y variadas.

Por el contrario, en el Perfil 1 casi todas las variables que componen el principio de caminar presentan valores positivos y estadísticamente significativos. La mayor parte de las áreas de estudio londinenses forman parte de este perfil. Sin embargo, el área London Bridge pertenece al Perfil 3, relativamente opuesto al Perfil 1 en cuanto a la caminabilidad se refiere. En la figura 6 se puede observar cómo el entorno construido alrededor de este importante nudo ferroviario presenta frecuentemente obras de reforma de gran envergadura, o al menos durante la toma de datos del estudio, lo que da lugar a la modificación de numerosos itinerarios peatonales, el aumento de los recorridos y las barreras, cambios en la señalización, etcétera, empeorando en este caso los valores alcanzados por los indicadores de caminabilidad. Esta circunstancia es similar a la de otras áreas del Perfil 3, como por ejemplo la de Plaza Egaña (Santiago), con obras que dificultan el acceso al nodo de transporte y grandes vías de circulación rodada alrededor del mismo.

En el Perfil 1 cabe reseñar, además, la existencia de bastantes áreas que disfrutan de valores elevados para el indicador 6.1 (densidad media comparada), como es el caso de Picadilly Circus, London Bridge, Aldgate (Londres), Plaza de la Ciudadanía (Santiago) o Metro Center (LA). Se trata de verdaderas "esquinas" urbanas (SoláMorales, 2004) donde estas ciudades adquieren y manifiestan morfológicamente funciones de representatividad, centralidad, pulso económico y escala (figura 6). Por estas razones, su densidad de uso del suelo está por encima de la media global.

FIgura 6 | London Bridge (izq.) y Picadilly Circus (der.), ambas en Londres, uK
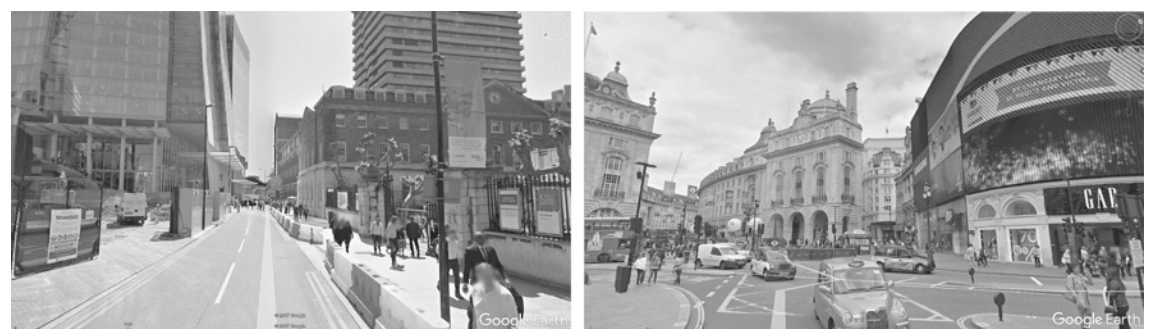

FUENTE GOOGLE STREET VIEW (2OI7)

\section{Análisis espacial}

El análisis SIG practicado pone de relevancia la importancia de llevar a cabo la espacialización de los resultados para comprender en profundidad la realidad urbana de las áreas de estudio (Serra-Coch, Chastel, Campos \& Coch, 2018). Para discutir este punto de vista, se contrastan a continuación las cartografías sIG obtenidas para las parejas de áreas: Gare Saint-Lazare - Opera y Gare Saint-Nazare - Vaugirard (figuras 3 y 4 , respectivamente).

Para las áreas Gare Saint-Lazare y Vaugirard, el reparto del valor medio correspondiente a la variable 1.3 es heterogéneo. Existe un elevado porcentaje de fachadas 
visualmente activas. En algunas ocasiones, las fachadas que no son visualmente activas se localizan en edificios públicos representativos, como por ejemplo la Estación de Ferrocarril de Saint-Lazare, la Ópera Garnier o la Parroquia Saint Lambert de Vaugirard. Se trata de edificios históricos, normalmente concentrados en conjuntos monumentales, que por sus características constructivas no presentan grandes huecos a fachada en planta baja. Modificar esta situación no sería tarea fácil, debido al grado de protección del planeamiento sobre ellos, y puede que tampoco deseable, por su notable presencia arquitectónica. En otras ocasiones se trata de grandes instalaciones (almacenes, uso industrial) cuya situación sería más fácil de revertir.

Igualmente, el reparto de la métrica 2.1 es escaso y tampoco es homogéneo. La distribución de calles con carriles-bicicletas seguros y completos no constituye una red completa, sino que presenta interrupciones entre unos tramos y otros, fundamentalmente en las intersecciones con otras calles. Esta circunstancia impide un aprovechamiento óptimo de la bicicleta como alternativa al transporte motorizado. La misma cuantía de ciclovía concentrada en torno al nodo de transporte formando una red conectada y continua mejoraría su rendimiento y posibilidades de uso. Para ello, el mapeo de la distribución espacial de esta variable mediante este tipo de análisis es muy útil.

Igualmente, la variable 3.1 para este ejemplo también presenta un reparto heterogéneo. Además, en ambas áreas existe una media de manzanas de gran longitud necesaria de tener en cuenta. Esta circunstancia introduce cierto desequilibrio a la hora de acceder a sendos nodos de transporte. En algunos frentes urbanos en torno a las mismas, constituidos por manzanas más pequeñas, este acceso es más favorable. Esto puede suponer ventajas de unas zonas urbanas sobre otras, como, por ejemplo, mediante el incremento de las rentas inmobiliarias (Cervero \& Landis, 1993).

El reparto de la métrica 1.5 en las áreas de Opera y Saint-Lazare no es homogéneo, pero sí eficaz. Pese a la baja puntuación obtenida en ambos casos, las zonas con árboles y las fachadas con porches cubiertos o toldos que producen sombra se concentran a lo largo de los espacios donde son más necesarios (avenidas principales, parques y plazas), como, por ejemplo, las arboledas del Boulevard Haussmann o los toldos de las boutiques de la Place Vendôme. Se trata de los espacios abiertos de mayor superficie, más desprotegidos frente al clima y de marcado uso público.

\section{Conclusiones}

Se ha comprobado que la Norma TOD es una herramienta sencilla para evaluar el nivel TOD de áreas construidas en torno a nodos de transporte, de forma global y útil en la mayoría de los casos, pero es limitada. Sirve para proporcionar una valoración general y resumida de cada área de estudio. Esto permite establecer un ranking de clasificación de áreas más o menos favorables al desarrollo orientado al transporte, lo que puede ser útil como certificación de calidad TOD. Sin embargo, más allá de esta puntuación, la información proporcionada es insuficiente para describir con precisión la complejidad del entorno urbano examinado.

El análisis multivariable som significa un paso más hacia adelante en el conocimiento de esta realidad. Permite una clasificación por perfiles de áreas más avanzada 
que la mera agrupación según Índice, determinando patrones de comportamiento afín de las distintas variables en cada asociación. Esta distinción de particularidades comunes permite encontrar similitudes desapercibidas para el método de evaluación basado en índices, lo que favorece a su vez criterios de actuación más pormenorizados. El método de evaluación mediante análisis som es, por tanto, más operativo en este sentido que el Índice TOD, más orientado al incentivo y clasificación general de niveles TOD por grupos de ciudades. Sin embargo, ambos métodos son complementarios, pues proporcionan información distinta que, correctamente interpretada, no es contradictoria.

Por otro lado, en ambos métodos la evaluación se realiza mediante valores medios, si bien es cierto que en el análisis som el Tamaño del Efecto (Es) y la significación estadística ayudan mucho a precisar los resultados. Aun así, esto se traduce en un problema clásico en el análisis estadístico de datos: la representatividad de los valores medios de las muestras estadísticas no tiene en cuenta la distribución de los datos alrededor de esta media. Dicho de otra forma, dos diferentes muestras estadísticas podrían tener la misma media, pero diferentes distribuciones. De ahí la importancia de otros parámetros estadísticos en la precisión de los resultados. El mapeo espacial mediante análisis SIG se ha verificado como una herramienta útil para precisar las diferentes distribuciones de valores en las áreas de estudio.

Visto desde el enfoque de la planificación, terapias de actuación comunes en áreas TOD con índices similares podrían suponer resultados distintos. En cambio, sí serían similares si se aplican en áreas del mismo perfil, al menos para los factores urbanos representados por variables estadísticamente significativas. Para saber con exactitud cómo y dónde intervenir dentro de estas áreas, heterogéneas por naturaleza, es necesario un mapeo espacial pormenorizado.

Una posible solución para que la evaluación mediante Índice ToD fuese más pormenorizada y efectiva, sería la subdivisión del área de estudio en múltiples zonas de menor tamaño. El objeto sería identificar con mayor precisión distribuciones irregulares de valores que contrarrestan la media y que, por lo tanto, no son representativas del área global. Estas subzonas, en consecuencia, deberían ser tratadas de forma distinta por el planeamiento urbano. La visión complementaria del planificador experimentado podría ayudar a realizar esa subdivisión de forma no sistemática o abstracta, siendo más atenta a factores caracterizadores del espacio urbano.

Los métodos de análisis exclusivamente cuantitativos o estadísticos son útiles para extraer conclusiones relevantes pero generales de la realidad urbana, puesto que tienden a homogeneizarla y simplificarla. Sin la intención de socavar sus ventajas, estos métodos se deben complementar con otras herramientas de corte espacial, de cara a mejorar su utilidad para la planificación y la habitabilidad en las ciudades. Estas herramientas permiten establecer una relación directa entre los valores obtenidos y la morfología urbana. Por esta razón, se convierten en la antesala del diseño urbano contemporáneo.

Los SIG son un ejemplo de este tipo de herramientas, aportando información extra a la cuantitativa-estadística. En nuestro caso de estudio, esta información se centra en: i) la localización espacial de los valores para cada indicador y su relación directa con la forma y elementos urbanos; ii) el grado de distribución espacial de 
estos valores dentro del área; iii) el grado de homogeneidad-heterogeneidad del área en cuanto a los valores obtenidos; y iv) la localización de las zonas que presentan carencias o excesos para cada variable. Además, realizan mediciones más precisas que otros métodos más convencionales, como los llevados a cabo en este trabajo para completar el cuestionario de la Norma TOD. Los resultados obtenidos mediante este análisis matizan y complementan la información proporcionada por otros métodos de evaluación, lo que ayuda a la planificación a la hora de elevar el nivel TOD del caso de estudio y similares.

\section{Referencias bibliográficas}

Abarca-Álvarez, F. J., Campos-Sánchez, F. S. \& Reinoso, R. (2017). Metodología de ayuda a la decisión mediante sig e Inteligencia Artificial: Aplicación en la caracterización demográfica de Andalucía a partir de su residencia. Estoa. Revista de la Facultad de Arquitectura y Urbanismo de la Universidad de Cuenca, 10(6), 33-51. https:// publicaciones.ucuenca.edu.ec/ojs/index.php/estoa/article/view/1433

Ackerson, K. J. (2005). A GIS approach to evaluating streetscape and neighborhood walkability (Master's thesis, Department of Planning, Public Policy and Management, and Graduate School of the University of Oregon). https://scholarsbank.uoregon.edu/ xmlui/handle/1794/1286

Baldwin, D. \& Lombardi, P. A. (2004). Policy support for and barriers to transit-oriented development in the inner city: Literature review. Transportation research record, 1887, 26-33. https://doi.org/10.3141/1887-04

Bação, F., Lobo, V. \& Painho, M. (2005). Self-organizing maps as substitutes for K-Means clustering. Computational Science-iccs 2005, 3516, 476-483. http://doi. org/10.1007/11428862_65

Bishop, Z. (2015). Transit-oriented development. Benefits and studies. Muncie, IN: Ball State University.

Carlthorpe, P. (1993). The next American metropolis: Ecology, community and the American dream. New York: Princeton Architectural Press.

Center for Transit-Oriented Development (Стор). (2009). Why transit-oriented development and why now? TOD 101. Oakland, CA: Reconnecting America and the Center for Transit-Oriented Development. http://www.reconnectingamerica.org/assets/Uploads/ tod101full.pdf

Cervero, R. (1998). The transit metropolis: A global inquiry. Wahington, DC: Island Press.

Cervero, R., Ferrell, C. \& Murphy, S. (2002). Transit-oriented development and joint development in the United States [Literature review of TCRP Project H-27, "TransitOriented Development: State of the Practice and Future Benefits"]. Transportation Research Record: Journal of the Transportation Research Board, 52. http://onlinepubs.trb. org/onlinepubs/tcrp/tcrp_rrd_52.pdf.

Cervero, R. \& Landis, J. (1993). Assessing the impacts of urban rail transit on local real estate markets using quasi-experimental comparisons. Transportation Research Part A: Policy and Practice, 27A(1), 13-22. https://doi.org/10.1016/0965-8564(93)90013-B 
Chanes, R. (2009). Deodendron. Árboles y arbustos de jardin en clima templado. Barcelona: Blume.

Coe, R. \& Merino, C. (2003). Magnitud del efecto: Una guía para investigadores y usuarios. Revista de Psicología, 21(1), 147-177. https://doi.org/10.1016/j.edumed.2017.07.002

Cohen, J. (1988). Statistical power analysis for the behavioral sciences. 2a ed. London: Lawrence Erlbaum Associates, Publishers. http://doi.org/10.1234/12345678

De Mattos, C. A. (1999). Santiago de Chile, globalización y expansión metropolitana: Lo que existía sigue existiendo. EURE, 25(76), 29-56. http://dx.doi.org/10.4067/S025071611999007600002

Dittmar, H. \& Ohland, G. (2004). The new transit town: Best practices in transit-oriented development. Washington, DC: Island Press.

Evans, J. E. \& Pratt, R. H., Stryker, A. \& Kuzmyak, J. R. (2007). Transit oriented development. En Transit Cooperative Research Program (TCRP) Report 95, Chap. 12. Washington DC: TCRP.

Fishman, R. (1987). Bourgeois utopias. The rise and fall of suburbia. New York: Basic Books.

García, C. (2004). Ciudad hojaldre: Visiones urbanas del siglo XXI. Barcelona: Gustavo Gili.

Hamaina, R., Leduc, T. \& Moreau, G. (2012). Towards urban fabrics characterization based on buildings footprints. En J. Gensel, D. Josselin \& D. Vandenbroucke (eds), Bridging the Geographic Information Sciences. Lecture Notes in Geoinformation and Cartography (pp. 231-248). Berlin, Heidelberg: Springer. https://doi.org/10.1007/978-3-64229063-3_18

Instituto de Políticas para el Transporte y el Desarrollo (The Institute for Transportation and Development Policy, ITDP) (2013). Desarrollo orientado al transporte. Regenerar las ciudades mexicanas para mejorar la movilidad. México, DF: ITDP. http://mexico.itdp. org/wp-content/uploads/Desarrollo-Orientado-al-Transporte.pdf

Instituto de Políticas para el Transporte y el Desarrollo (The Institute for Transportation and Development Policy, ITDP) (2014). The TOD Standard v2.1. Nueva York. https://www. itdp.org/tod-standard/

Jacobson, J. \& Forsyth, A. (2008). Seven American tops: Good practices for urban design in transit-oriented development projects. Journal of transport and land use, 1(2), 51-88. https://doi.org/10.5198/jtlu.v1i2.67

Kaski, S. \& Kohonen, T. (1996). Exploratory data analysis by the self-organizing map: structures of welfare and poverty in the world (1996). Neural networks in financial engineering. Proceedings of the Third International Conference on Neural Networks in the Capital Markets (pp. 498-507). https://doi.org/10.1.1.53.3954

Kauko, T. (2005). Using the self-organising map to identify regularities across country-specific housing-market contexts. Environment and Planning B: Planning and Design, 32(1), 89-110. https://doi.org/10.1068/b3186

Leslie, E., Coffee, N., Frank, L., Owen, N., Bauman, A. \& Hugo, G. (2007). Walkability of local communities: Using geographic information systems to objectively assess relevant environmental attributes. Health \& Place, 13, 111-122. https://doi: 10.1016/j. healthplace.2005.11.001

Newman, P. \& Kenworthy, J. (1999). Sustainability and cities: Overcoming automobile dependence. Washington, DC: Island Press. 
Renne, J. L. \& Wells, J. S. (2002). State of the literature: Transit-oriented development. Assessing the impacts of the New Jersey transit village initiative. New Brunswick, NJ: Rutgers. The State University of New Jersey.

Renne, J. L. \& Wells, J. S. (2005). Research results digest 294. Resumen de los principales hallazgos del proyecto "Transit oriented development: Developing a strategy to measure success", dirigido por J. L. Renne y J. S. Wells. National Cooperative Highway Research Program, February 2005. https://www.nap.edu/read/23319/chapter/1

Rosas, J. (1985). La partición de la manzana: cómo se modernizó Santiago de Chile. Revista UR, 3, 29-38. http://hdl.handle.net/2099/2992

Sassen, S. (2003). Localizando ciudades en circuitos globales. EURE, 29(88), 5-27. http:// dx.doi.org/10.4067/S0250-71612003008800001

Sassen, S. (2013). The Global City: New York, London, Tokyo. Princeton, NJ: Princeton University Press.

Schlossberg, M. \& Brown, N. (2004). Comparing transit-oriented development sites by walkability indicators. Transportation Research Record, 1887, 34-42. https://doi. org/10.3141/1887-05

Serra-Coch, G., Chastel, C., Campos, S. \& Coch, H. (2018). Graphical Approach to assess urban quality: Mapping walkability based on the ToD-standard. Cities, 76, 58-71. https://doi.org/10.1016/j.cities.2018.01.007

Silver, M. S. (2008). On the design features of decision support systems: The role of system restrictiveness and decisional guidance. En F. Burstein \& C. W. Holsapple (eds.), Handbook on Decision Support Systems 2: Variations (pp. 261-291). Berlín / Heidelberg: Springer-Verlag.

Singh, Y. J., Fard, P., Zuidgeest, M., Brussel, M. \& van Maarseveen, M. (2014). Measuring transit oriented development: A spatial multi criteria assessment approach for the City Region Arnhem and Nijmegen. Journal of Transport Geography, 35, 130-143. https:// doi.org/10.1016/j.jtrangeo.2014.01.014

Skupin, A. \& Agarwal, P. (2008). Introduction: What is a self-organizing map? En P. Agarwal \& A. Skupin (eds.), Self-organising maps: Applications in geographic information science (pp. 1-20). Hoboken, NJ: Wiley.

Soja, E. W. (2001). Postmetropolis: Critical studies of cities and regions. Oxford: Blackwell.

Solá-Morales, M. (2004). Ciudades, esquinas. Foro universal de las culturas. Bitácora Arquitectura, 13, 28-37. http://dx.doi.org/10.22201/fa.14058901p.2005.13.26288

Spielman, S. E. \& Thill, J. C. (2008). Social area analysis, data mining, and GIS. Computers, Environment and Urban Systems, 32(2), 110-122. https://doi.org/10.1016/j. compenvurbsys.2007.11.004

Suzuki, H. Cervero, R. \& Iuchi, K. (2013). Transforming cities with transit. Transit and land-use integration for sustainable urban development. Urban development series. Washington, DC: World Bank. http://documents.worldbank.org/curated/ en/947211468162273111/Main-report

Turán, N. (2011). Towards an ecological urbanism for Istanbul. En A. Sorensen \& J. Okata (eds.), Megacities. Library for Sustainable Urban Regeneration, Tokio: Springer (vol. 10, pp. 223-243). https://doi.org/10.1007/978-4-431-99267-7_10 
Wasserstein, R. L. \& Lazar, N. A., 2016. The AsA's statement on $p$-Values: context, process, and purpose. The American Statistician, 70(2), 129-133. http://doi.org/10.1080/0003130 5.2016 .1154108

Weiss, S. \& Indurkhya, N. (1997). Predictive Data Mining: A Practical Guide. San Francisco, CA: Morgan Kaufmann Publishers.

Yeh, A. (2005). Urban planning and gis. En P. A. Longley, M. F. Goodchild, D. J. Maguire \& D. W. Rhind (eds.), Geographical Information Systems: Principles, techniques, management and applications [Abridged, $2^{\text {nd }}$ ed.], (pp. 877-888). Hoboken, NJ: John Wiley \& Sons. http://www.geos.ed.ac.uk/_gisteac/gis_book_abridged/files/ch62.pdf

Zhang, J., Shi, H. \& Zhang, Y. (2009). Self-organizing map methodology and google maps services for geographical epidemiology mapping. Conference Paper. DICTA 2009. Digital Image Computing: Techniques and Applications (pp. 229-235). http://doi. org/10.1109/DICTA.2009.46 